\title{
Perfil Psicológico de Migrantes Através da Forma IRF do MMPI'
}

\author{
Valéria Barbieri² e André Jacquemin \\ Universidade de São Paulo, Ribeirão Preto
}

\begin{abstract}
RESUMO - Devido à associação freqüentemente apontada na literatura entre o fenômeno da migração e o desenvolvimento de distúrbios mentais, este trabalho tencionou fornecer o perfil psicológico de uma amostra de migrantes, por meio de uma forma reduzida de um instrumento de valor clínico e empírico comprovado: o MMPI. Para tanto, 20 migrantes do sexo masculino, analfabetos e sem comprometimentos intelectuais severos foram submetidos à forma IRF do MMPI, desenvolvida nos Estados Unidos em 1980, destinada a sujeitos com essas características cognitivas. Os resultados se constituíram em altas pontuações na escala $F$ de validade, relacionadas principalmente a questões de ordem cultural e incompreensão dos itens. Em termos clínicos, dentre os protocolos considerados confiáveis para análise, foi detectada uma tendência da IRF a produzir elevações nos resultados das escalas clínicas de um modo generalizado, indicando problemas relativos à padronização, validade e capacidade discriminativa do instrumento para uso na população brasileira não alfabetizada.
\end{abstract}

Palavras-chave: MMPI; IRF; migração; analfabetos; psicopatologia.

\section{Migrants’ Psychological Profiles From MMPI Improved Readability Form}

\begin{abstract}
Due to the association, frequently pointed out by literature, between the phenomenon of human migration and the development of mental disturbances, this work intends to supply the psychological profile of a migrants ' Brazilian sample, by means of a reduced form of an instrument which has had its clinical and empirical value proved: the MMPI. For this, 20 masculine migrants, illiterates and without severe intellectual handicaps were submitted to IRF form of MMPI, that was developped in the USA for individuals with those cognitive characteristics. The results showed high scores in the $\mathrm{F}$ scale, related mainly to cultural issues and misunderstanding of items. Among the protocols that seem to be reliable for analysis have been detected a general IRF tendency of producing high scores in clinical scales. This indicates difficulties related to IRF standartization, validity and discrimination used for the Brazilian illiterate population.
\end{abstract}

Key words: MMPI; IRF; migration; illiterates; psychopatology.

Dentre as variáveis freqüentemente estudadas pela Epidemiologia Psiquiátrica, aquela que mais tem chamado a atenção de pesquisadores, principalmente na América do Sul, é o fenômeno da migração, evento paralelo à rápida urbanização dos países do terceiro mundo. O motivo da atenção dos pesquisadores ter se focalizado nesse fenômeno deve-se à dimensão que ele tem alcançado nas últimas décadas.

Compartilhando dessa tendência, a população urbana no Brasil de acordo com o censo de 1970 foi estimada em torno de $56 \%$, contra $67,5 \%$ no recenseamento de 1980 (Almeida Filho, 1986), 75,6\% em 1991 e 78,4\% em 96. Na década de 80 , o número de migrantes foi estimado em mais de $11 \mathrm{mi}-$ lhões. Ainda, durante o período entre 1991 e 1996, mais de 10 milhões de pessoas apresentaram história de migração.

1 O presente artigo é resultado do trabalho de Dissertação de Mestrado de Valéria Barbieri, desenvolvida junto ao programa de pós-graduação em Psicologia Clínica do Instituto de Psicologia da Universidade de São Paulo (IPUSP). Os autores remetem agradecimentos ao CNPq pelo suporte financeiro à primeira fase da pesquisa e à Universidade de Franca pelo apoio à segunda. Agradecimentos também são realizados à Central de Encaminhamento do Migrante e Mendicante (CETREM) da cidade de Ribeirão Preto, que permitiu a coleta de dados.

2 Endereço: Faculdade de Filosofia, Ciências e Letras de Ribeirão Preto - USP, Av. Bandeirantes 3900, Campus Universitário, Monte Alegre CEP: 14040-901, Ribeirão Preto-SP. E-mail: valeriab@ffclrp.usp.br ou vbarbieri@netsite.com.br
Nesse sentido, Chaimowicz (1997) assevera que o movimento migratório, principalmente de jovens procedentes das regiões norte e nordeste com destino à sudeste, vem acarretando importantes modificações na dinâmica populacional dessas áreas, em particular relacionadas à proporção da população idosa nelas presente e à taxa de natalidade. Contudo, o mesmo autor observa que o censo de 1991 indica uma tendência à redução das correntes migratórias nordestinas em direção ao sudeste, com retenção das populações nas capitais e cidades de médio porte dos próprios estados de origem.

Em termos de Psicologia e Psiquiatria, vários estudos no Brasil e no exterior têm demonstrado a existência de uma associação entre o fenômeno da migração e o desenvolvimento de distúrbios mentais.

Seguín (conforme citado por Almeida Filho, 1985) realizou um estudo no Peru em 1956, onde foi encontrada alta prevalência de sintomas psicossomáticos (relativos aos aparelhos circulatório, gastrointestinal, respiratório e neurológico), além de depressão e ansiedade em índios montanheses que haviam migrado recentemente para a cidade.

Rotondo (também citado por Almeida Filho, 1985) comparando os resultados de migrantes e não migrantes no Cornell Medical Index, encontrou maior prevalência de epilepsia, reações depressivas e desordens psicossomáticas entre os primeiros e psicoses e reações de ansiedade entre os últimos. 
Tratando do fenômeno da imigração, Cochrane e Bal (1987) examinaram as taxas de admissão psiquiátrica por esquizofrenia na Inglaterra, encontrando maior número dessa atribuição diagnóstica entre imigrantes paquistaneses do sexo masculino, irlandeses, indianos e caribenhos. Johanson, Sundquist, Johanson e Bergman (1998) por sua vez, comparando as taxas de admissão psiquiátrica entre nativos da Suécia e pessoas que imigraram para esse país não encontraram diferenças significativas nesse sentido. Contudo, verificaram que, considerando a amostra total, o risco de readmissão psiquiátrica aumentava significativamente entre os sujeitos que apresentavam altas taxas de migração interna.

Sam e Berry (1995) estudando crianças procedentes da Ásia, África, América do Sul e Turquia que haviam emigrado para a Noruega, não encontraram uma relação significativa entre o status de imigrante em si e o desenvolvimento de transtornos emocionais. Contudo, ao relacionar tais distúrbios com a experiência e atitudes perante a aculturação, variáveis demográficas e interpessoais, verificaram a existência de um relacionamento sistemático subjacente entre estes fatores e a existência do stress de aculturação. Também referindo-se à relevância do processo de aculturação para o desenvolvimento da saúde ou enfermidade mental, Escobar (1998) discute o estudo realizado por Vega, Kolody, Hwang e Noble (1993) no qual foram encontrados melhores níveis de saúde mental em mexicanos que emigraram para os Estados Unidos do que em seus descendentes nascidos nesse país. Segundo Escobar, esses resultados poderiam ser explicados em razão dos imigrantes mexicanos apresentarem mecanismos de proteção das tradições originais, o que teria um efeito positivo sobre a saúde mental, enquanto o processo da aculturação em si, seria pernicioso nesse sentido.

No Brasil, Almeida Filho e Bastos (1982) comparando prontuários psiquiátricos de pacientes femininos com síndrome depressiva (incluindo quadros de reação depressiva, neurose depressiva, hipocondria e psicose maníaco depressiva) com dados de um inquérito de prevalência de doenças mentais realizado na cidade de Salvador, verificaram que o risco de uma mulher migrante desenvolver tais sintomas é 2,5 vezes maior do que uma não migrante.

No mesmo ano, Santana (conforme citada por Almeida Filho, 1985), aplicando o QMPA (Questionário de Morbidade Psiquiátrica para Adultos) em indivíduos residentes em um bairro pobre de Salvador, encontrou maior prevalência de desordens mentais em migrantes, principalmente neuroses. Souza e Silva (1998) em um estudo sobre os riscos de desenvolvimento de distúrbios psiquiátricos menores em motoristas e cobradores de ônibus urbanos da cidade de São Paulo, encontraram maior prevalência desses transtornos em sujeitos que haviam migrado da região nordeste.

Embora haja praticamente unanimidade na literatura quanto à relação entre o status de migrante e a maior possibilidade de desenvolver distúrbios psíquicos, existem alguns embates na Epidemiologia referentes tanto à definição de migração em si, por que e como esse fenômeno é capaz de determinar ou desencadear distúrbios mentais, metodologias de pesquisa utilizadas e técnicas diagnósticas empregadas.
Com relação às técnicas diagnósticas, de acordo com a revisão efetuada por Almeida Filho (1985), há uma diversidade tal na natureza dos instrumentos empregados que vai desde técnicas antropológicas, observação e conversação, até testes psicológicos altamente estruturados como o Cornell Medical Index, passando pelo TAT, escalas isoladas de personalidade e questionários construídos pelos próprios autores das pesquisas.

Essa diversidade muitas vezes responde ao próprio referencial teórico-filosófico da pesquisa (antropológico, clínico ou estatístico) e, embora proporcione o enriquecimento característico dos diferentes olhares sobre o mesmo tema, acarreta uma conseqüência bastante importante, a saber, os diferentes níveis de cuidado na escolha dos instrumentos.

Dentre as técnicas mais populares empregadas nos estudos epidemiológicos encontra-se o Cornell Medical Index (CMI) que, embora se constitua numa medida objetiva de fácil e rápida aplicação, é bastante sensível à simulação ou dissimulação por parte do examinando.

A literatura sobre as técnicas de exame psicológico aponta como instrumento passível de substituir com superioridade o CMI na atividade diagnóstica, o Inventário Multifásico Minnesota de Personalidade (MMPI).

Mesmo apresentando uma série de vantagens (construção empírica, escalas de confiabilidade, validade e precisão comprovadas) o MMPI tem suas limitações. A primeira delas refere-se à extensão do teste: a exigência de responder 566 questões consome no mínimo uma hora e meia de trabalho do aplicador e do examinando. Essa dificuldade vem sendo contornada pelo desenvolvimento de formas reduzidas do inventário, as quais vêm se mostrando como relativamente promissoras na tarefa de substituir o instrumento-padrão.

Outra limitação importante é a de que, como instrumento verbal, o MMPI é bastante sensível aos efeitos da variável escolaridade. De fato, Johnson e Bond em 1950 definiram a sexta série do sistema de ensino norte-americano (ou oitava série do brasileiro) como o limite mínimo de instrução exigido para um sujeito completar o MMPI. Esse limite foi revisto por Blanchard em 1981, que estabeleceu o critério de 10 anos de escolaridade para que um sujeito pudesse compreender de 90 a $100 \%$ dos itens do inventário. Esta restrição é particularmente relevante no Brasil, onde 23,3\% da população sequer chega a ser alfabetizada.

Visando diluir o constrangimento acadêmico imposto pelo MMPI e, ao mesmo tempo, restringir o tempo de aplicação do teste, Ward e Selby (1980) desenvolveram nos Estados Unidos uma forma reduzida do inventário destinada a sujeitos não alfabetizados, com habilidades verbais prejudicadas, nível de leitura empobrecido ou apresentando deficiências no plano cognitivo.

Nesse processo, elaboraram uma forma reduzida composta por 167 itens que são administrados verbalmente aos sujeitos. Ward e Selby denominaram sua redução de IRF (Improved Readability Form). Avaliando o grau de instrução escolar requerido pela IRF, os autores concluíram que o 
instrumento ainda solicitava a sexta série como limite mínimo, enquanto o MMPI completo exigiria a sétima série. Não obstante, os mesmos autores afirmam que, em sua pesquisapiloto a IRF, quando aplicada oralmente, demonstrou-se compreensível por indivíduos com QI até 65.

Comparados os resultados fornecidos pela IRF com aqueles do MMPI, foram encontrados níveis de correlação que variaram de 0,83 a 0,96 (Ward \& Selby, 1980), 0,82 a 0,96 (Ward, 1986) e 0,68 a 0,96 (Ward \& Meyers, 1984).

Observadas as características da forma IRF pode-se antever que, caso comprovado seu valor diagnóstico e grau de correspondência com o MMPI, tratar-se-ia de um instrumento bastante promissor, tanto do ponto de vista clínico como epidemiológico. Assim, este trabalho visa contribuir com a pesquisa epidemiológica em Psicologia, avaliando a qualidade das informações trazidas pela IRF na compreensão do psiquismo de migrantes brasileiros não alfabetizados. Como segundo objetivo, tenciona-se realizar um levantamento epidemiológico das características de personalidade desse grupo, contrapondo-as às assinaladas na literatura.

\section{Metodologia}

\section{Sujeitos}

Participaram da pesquisa 20 migrantes do sexo masculino e de baixo nível sócio-econômico, que procuravam assistência social em uma instituição municipal da cidade de Ribeirão Preto. Todos os sujeitos eram analfabetos segundo o critério do IBGE $(1980,1991,1997)$ e $95 \%$ se encontravam desempregados. Cerca da metade do grupo procedia da zona rural; a idade variou entre 18 e 64 anos. Setenta por cento dos sujeitos eram solteiros e não referiam antecedentes psicopatológicos.

\section{Material}

Foram utilizados conjuntos do Teste de Inteligência Não Verbal (INV) - forma C, desenvolvido por Pierre Weil em 1951 (conforme citado por Weil \& Nick, 1971), além de cadernos de teste contendo os itens componentes da IRF. Empregou-se ainda um glossário contendo termos constituintes do teste de personalidade, de modo a possibilitar o esclarecimento de dúvidas relativas ao significado de palavras específicas.

\section{Procedimento}

Após o estabelecimento do "rapport" o sujeito era submetido à aplicação individual do INV - forma C, com o intuito de detectar possíveis comprometimentos intelectuais que pudessem interferir na compreensão dos itens da IRF. A opção pelo emprego de um instrumento de avaliação intelectual não verbal nesse contexto, realizou-se em função da inexistência de materiais verbais passíveis de aplicação à população brasileira não alfabetizada. Finda a avaliação intelectual, o examinando dispunha de um intervalo de tempo para descanso antes de submeter-se à avaliação de personalidade. A administração da IRF foi realizada individualmente e verbalmente. Nas situações em que o sujeito apresentava dúvidas quanto ao significado de palavras ou expressões específicas, havia o esclarecimento de acordo com os sinônimos do glossário. Dúvidas referentes à compreensão global dos itens não foram elucidadas.

\section{Resultados}

De acordo com os dados fornecidos pelo Teste de Inteligência Não Verbal - forma C, a faixa de variação do potencial intelectual dos migrantes estendeu-se de infradotado a superdotado. Apesar da amplitude de variação, 90\% dos sujeitos (18) obtiveram diagnósticos intelectuais médio e acima da média. Quanto aos 2 sujeitos restantes, um foi classificado como intelectualmente médio-inferior e o outro como infradotado. As normas do INV utilizadas referem-se às apresentadas por Weil e Nick (1971) para indivíduos não alfabetizados.

No que se refere à análise das escalas de validade da IRF, a distribuição dos perfis quanto à sua confiabilidade, é exposta a seguir.

A observação da Tabela 1 indica que cerca de dois terços dos sujeitos não abordaram a tarefa do modo como pretendiam os autores do teste, o que coloca os resultados obtidos nas escalas clínicas sob suspeita. A quantidade elevada de protocolos nessas condições exigiu investigações mais profundas acerca do modo como ocorreram as invalidações. Assim, a Tabela 2 mostra o número de pontuações $T \geq 70$ em cada escala de validade.

O exame da Tabela 2 indica que a principal determinante das invalidações constituiu-se em $F$ : metade dos migrantes obtiveram pontuações maiores ou iguais a $70 \mathrm{~T}$ nessaescala. A significação interpretativa dessas elevações, segundo Dahlstrom e Welsh (1968), Hathaway e McKinley (1971), Mendonça, Elliot, McNeill, Rodriguez e Zelkind (1984), Cunha, Freitas e Raymundo (1986) e Graham (1977/1987), abrange tanto modalidades de conduta do sujeito perante a prova, quanto traços psicopatológicos e de personalidade. Uma das interpretações usualmente atribuídas ao alto esco-

Tabela 1. Número de protocolos validados e invalidados

\begin{tabular}{lc}
\hline Tipos de protocolo & Número de sujeitos \\
\hline Validados & 7 \\
Invalidados & 13 \\
\hline Total & 20 \\
\hline
\end{tabular}

Tabela 2. Número de escores $T \geq 70$ em cada escala de validade

\begin{tabular}{cc}
\hline Escala de Validade & Número de invalidações \\
\hline$?$ & 1 \\
L & 4 \\
F & 10 \\
K & 1 \\
\hline Total $^{3}$ & 16 \\
\hline
\end{tabular}

3 O total exposto ultrapassa o referente ao número de protocolos invalidados (quadro 1) pois o mesmo sujeito pode alcançar pontuação $T \geq$ 70 em mais de uma escala. 
re em $F$ diz respeito à possibilidade de incompreensão dos itens devido a limitações intelectuais. Embora a grande maioria dos sujeitos não apresentasse comprometimentos cognitivos segundo indicado pelo INV, procedeu-se à verificação se essas invalidações estariam associadas ao nível intelectual. A aplicação do Teste Qui-quadrado comparando o número de invalidações por $F$ nas categorias intelectuais inferiores à média e média ou superiores demonstrou que a pontuação $T \geq 70$ não se associava ao potencial cognitivo dos migrantes ( $p=0,2314$ numa prova unilateral), indício que apontaria para a confiabilidade dos perfis. No entanto, há que se considerar que esta avaliação cognitiva restringiuse à determinação do nível intelectual não verbal dos sujeitos. Embora a literatura admita a equivalência entre o potenciais cognitivos verbal e não verbal de um mesmo indivíduo (Pichot, 1961; Raven, 1950; Saraiva ,1961) sendo esse o pressuposto que viabiliza a avaliação da deterioração intelectual, há algumas incursões na bibliografia que supõem não ser sempre esse o caso. Raven (1950) tratando da equivalência diagnóstica da Escala Geral das Matrizes Progressivas e do Teste de Vocabulário de Mill Hill, comenta que eventualmente, quando um examinando apresenta resultados mais altos nas primeiras que no segundo, pode-se inferir que ele não obteve as informações e o domínio da linguagem que sua capacidade intelectual lhe permite. Wechsler (conforme citado por Saraiva, 1961) afirma que:

Contrariamente à opinião leiga, a extensão do vocabulário de um indivíduo não é apenas um índice de sua escolaridade, mas também uma excelente medida de sua inteligência geral (...) A única objeção séria que pode ser feita contra a utilização do teste de vocabulário como teste de inteligência, é que o número de palavras que um sujeito adquire deve ser necessariamente influenciado por seu ambiente educacional e cultural. Ele é aparentemente inadequado para indivíduos incultos e estrangeiros.

Portanto, embora os resultados obtidos no INV assegurem a seleção de uma amostra livre de comprometimentos intelectuais severos, é arriscado transpor o diagnóstico obtido por meio desse instrumento para a esfera verbal, uma vez que a experiência escolar dos sujeitos é mínima ou inexistente.

Nesse sentido, descartou-se na análise clínica da IRF, os protocolos cujas pontuações eram iguais ou maiores que $70 T$ em uma ou mais escalas de validade (incluindo os referentes aos dois sujeitos identificados pelo INV como intelectualmente abaixo da média) a fim de assegurar maior confiabilidade dos resultados. Com isso, dos 20 sujeitos iniciais, restaram apenas 7 protocolos passíveis de análise.

O número de sujeitos segundo os níveis de pontuação nas escalas clínicas é ilustrado a seguir.

O exame da Tabela 3 mostra uma concentração de sujeitos apresentando pelo menos uma escala clínica com pontuação $\geq 70 T(57,14 \%)$; na faixa de elevações moderadas $(60$ $\leq T \leq 69)$ encontra-se o restante do grupo $(42,85 \%)$.

A Tabela 4 ilustra a distribuição dos sujeitos pela quantidade das escalas com pontuação $T \geq 70$ em seus perfis.

A observação da Tabela 4 permite afirmar que, embora a maioria dos sujeitos apresente pontuação $T \geq 70$ em alguma escala clínica, não se pode definir uma tendência relativa à quantidade delas por protocolo; pelo contrário, a distribuição é dispersa. Dado que a amostra dispõe de um número bastante reduzido de componentes, é impossível concluir se essa dispersão aplica-se ou não ao grupo de migrantes como um todo.

Pormenorizando essas informações, a Tabela 5 expõe a distribuição das pontuações em cada uma das escalas clínicas.

Como já era possível antever pelas Tabelas 3 e 4, os escores alcançados pelos migrantes concentraram-se nas faixas elevada $(\geq 70 \mathrm{~T})$ e moderadamente elevada $(60 \leq T \leq$ 69): estas duas categorias compreendem 38 dos 70 escores do grupo (54\%). Por outro lado, é curioso observar que há poucas pontuações baixas: apenas $15 \%$ dos escores são iguais ou inferiores a $49 T$ e nenhum é menor que 39T. A análise da Tabela 5 permite verificar que nenhuma das escalas clínicas se destaca em relação às outras por apresentar uma concentração maior de pontuações elevadas ou moderadamente elevadas. De fato existe uma nova dispersão dos resultados, indicando que a IRF não aponta nenhuma tendência parti-

Tabela 3. Número de sujeitos com pelo menos uma escala clínica incluída em cada faixa de pontuação $T$

\begin{tabular}{cc}
\hline Pontuações $\boldsymbol{T}$ & Número de sujeitos \\
\hline$\geq 70$ & 4 \\
$60-69$ & 3 \\
$<60$ & 0 \\
\hline Total & 7 \\
\hline
\end{tabular}

Obs. As faixas são mutuamente exclusivas, priorizando as pontuações mais altas.

Tabela 4. Número de sujeitos de acordo com a quantidade de escalas clínicas com pontuações $T \geq 70$

\begin{tabular}{|c|c|}
\hline Quantidade de escalas com pontuação $T \geq 70$ & Número de sujeitos \\
\hline 0 & 3 \\
\hline 1 & 1 \\
\hline 2 & 0 \\
\hline 3 & 1 \\
\hline 4 & 0 \\
\hline 5 & 0 \\
\hline 6 & 2 \\
\hline Total & 7 \\
\hline
\end{tabular}

Tabela 5. Freqüência de escores por categoria de pontuação em cada escala clínica

\begin{tabular}{lcccccc}
\hline $\begin{array}{l}\text { Escalas } \\
\text { Clínicas }\end{array}$ & $\boldsymbol{T} \geq \mathbf{7 0}$ & $\mathbf{6 0}$ a $\mathbf{6 9 T}$ & $\mathbf{5 0}$ a $\mathbf{5 9 T}$ & $\mathbf{4 0}$ a $\mathbf{4 9 T}$ & $\boldsymbol{T} \leq \mathbf{3 9}$ & Total \\
\hline $\mathrm{Hs}$ & 2 & 2 & 2 & 1 & 0 & 7 \\
$\mathrm{D}$ & 2 & 3 & 2 & 0 & 0 & 7 \\
$\mathrm{Hy}$ & 2 & 0 & 2 & 3 & 0 & 7 \\
$\mathrm{Pd}$ & 2 & 2 & 2 & 1 & 0 & 7 \\
$\mathrm{M} \mathrm{f}$ & 2 & 2 & 2 & 1 & 0 & 7 \\
$\mathrm{~Pa}$ & 1 & 3 & 2 & 1 & 0 & 7 \\
$\mathrm{Pt}$ & 2 & 2 & 2 & 1 & 0 & 7 \\
$\mathrm{Sc}$ & 2 & 2 & 2 & 1 & 0 & 7 \\
$\mathrm{M} \mathrm{a}$ & 2 & 3 & 2 & 0 & 0 & 7 \\
$\mathrm{Si}$ & 1 & 1 & 3 & 2 & 0 & 7 \\
\hline Total & 18 & 20 & 21 & 11 & 0 & 70 \\
\hline
\end{tabular}


cular em relação a traços de personalidade que caracterizariam essa amostra de sujeitos.

Como complemento a essas informações a Tabela 6 , mostra que os escores médios em cada escala variaram de $T=55$ a $T=65,57$. As menores pontuações foram obtidas nas escalas $\mathrm{Si}$ e $\mathrm{Hy}$ e as maiores em $P d$ e $M a$ (escalas componentes da tétrade psicótica do MMPI).

\section{Discussão}

Os resultados encontrados permitem realizar algumas considerações quanto às possibilidades de emprego da IRF em migrantes brasileiros não alfabetizados, sem a pretensão de apresentar um caráter conclusivo devido à reduzida dimensão da amostra.

Inicialmente há que se questionar a validade do emprego do Teste INV - forma C - como preditor da capacidade do examinando entender os itens da IRF, uma vez que muitos dos sujeitos classificados como apresentando nível intelectual médio ou superior tiveram seus protocolos invalidados pela escala $F$, indicadora de incompreensão. Essa constatação aponta para duas questões que necessitam ser investigadas. A primeira delas refere-se à validação do INV - forma $\mathrm{C}$ para a população brasileira não alfabetizada, uma vez que para a amostra em questão, esse instrumento revelou-se como bastante fácil e não discriminativo do desempenho dos sujeitos em uma tarefa posterior. Portanto, seriam necessárias investigações acerca da validade preditiva desse material, bem como novos estudos de padronização para a população a que se dirige. A segunda questão diz respeito ao estudo da relação entre os níveis intelectuais verbais e não verbais de indivíduos analfabetos, visto que, como já apontava Wechsler (citado por Saraiva em 1961) e Raven (1950) não se pode simplesmente transpor para esses sujeitos conclusões oriundas de estudos com pessoas apresentando outros graus de instrução. Nesse ponto, seria interessante a confecção de um material verbal padronizado destinado à avaliação da população analfabeta, visando fins como os da presente pesquisa.

No que se refere aos resultados obtidos na IRF, verificase uma tendência geral à elevação dos escores das escalas clínicas. Mesmo dentre os protocolos considerados válidos, encontra-se uma concentração das pontuações nas faixas elevada e moderadamente elevada, sendo que todos os su-

Tabela 6. Médias e desvios-padrões dos escores de cada escala clínica da IRF

\begin{tabular}{ccc}
\hline Escalas Clínicas & Escore médio & Desvio-padrão \\
\hline Hs & 60,29 & 10,42 \\
D & 57,43 & 9,52 \\
Hy & 56,29 & 12,82 \\
Pd & 65,57 & 14,82 \\
Mf & 62,71 & 11,18 \\
Pa & 61,29 & 9,57 \\
Pt & 62,00 & 11,49 \\
Sc & 63,14 & 12,93 \\
Ma & 64,71 & 10,23 \\
Si & 55,00 & 9,85 \\
\hline
\end{tabular}

jeitos obtiveram ao menos uma escala nessas faixas de escores. Tal observação, acrescida ao fato de existir uma dispersão de resultados elevados nas diferentes escalas clínicas, não permite a indicação de nenhuma tendência grupal como afirma a literatura, por exemplo, a presença de distúrbios psicossomáticos apontada por Seguín e Rotondo (conforme citados por Almeida Filho, 1985) ou a existência de maiores taxas de esquizofrenia como indicada por Cochrane e Bal (1987). No máximo, os resultados permitiriam confirmar as conclusões de Santana (conforme citada por Almeida Filho, 1985), que encontrou piores níveis de saúde mental em migrantes residentes em Salvador, e de Souza e Silva (1998) que revelaram maior risco de desenvolvimento de distúrbios psiquiátricos em motoristas e cobradores de ônibus apresentando história de migração. Porém, a constelação dos resultados fornece, antes de tudo, um forte subsídio à hipótese de que os escores alcançados refletem a parcialidade cultural da técnica de avaliação ao invés de indicar características psicológicas dos sujeitos. Nesse sentido, qualquer interpretação do perfil psicológico obtido pela amostra seria arriscada. Em suma, há indícios de que o emprego eficaz da forma IRF do MMPI em indivíduos analfabetos brasileiros requer ainda alguns procedimentos de simplificação dos itens de modo a torná-los mais compreensíveis, mas, sobretudo, há necessidade premente de pesquisas de padronização e validação do material para essa população, além do aperfeiçoamento de sua capacidade discriminativa, que mostrou-se precária.

Nesse momento há que se recordar que Hathaway e McKinley (1971) em seu manual do MMPI afirmam que uma das causas para a elevação de escores na escala $F$ é a não participação do sujeito na estrutura cultural comum à redação dos itens.

Uma análise mais demorada das proposições componentes da escala $F$ indica que efetivamente os sujeitos apresentam algumas respostas inusitadas que parecem refletir vivências e crenças culturais específicas, como por exemplo na resposta "V" (Verdadeiro) ao item "Às vezes maus espíritos se apoderam de mim", "F" (Falso) a "Creio em outra vida depois desta" e a "Deve-se ensinar às crianças todos os fatos principais da vida sexual". O item "Meu pai foi um bom homem" respondido "F" por vários sujeitos assentouse numa base real para eles, que haviam sido abandonados pelo pai na infância.

Portanto, a alta taxa de invalidação pela escala $F$ encontrada neste trabalho parece dever-se à atuação conjunta de incompreensão e incompatibilidade cultural com a amostra que serviu de parâmetro para a definição das normas brasileiras do MMPI. Dado que existe correlação entre os resultados de $F$ e o das escalas indicadoras de características psicóticas do MMPI, os escores médios mais elevados em $P d, M a$ e $S c$ conforme apontados na Tabela 6 , também podem ser decorrentes das mesmas causas.

Não é possível, contudo, ignorar que certos itens da escala $F$ respondidos insolitamente apresentam caráter patológico mais explícito como "Minha vida sexual é satisfatória" ("F"), "A maior parte do tempo preferiria sentar-me e deva- 
near a fazer qualquer outra coisa" ("V"), "Alguém anda tentando envenenar-me" ("V"), entre outros.

Embora algumas dessas proposições tenham-se mostrado suscetíveis de incompreensão (por exemplo, vários sujeitos desconheciam o significado do termo "devanear") não se pode descartar por completo a hipótese da existência de dificuldades psicológicas. Nesse sentido, é importante destacar que alguns dos sujeitos referiram idéias ou tentativas de suicídio à examinadora durante a aplicação do material.

De qualquer maneira, devido às dificuldades já discutidas referentes à padronização, validade e capacidade discriminativa da técnica, é impossível discernir por meio deste experimento, onde termina a influência, nos resultados, da incompatibilidade cultural e começa a indicação de real psicopatologia. Assim, uma das conclusões possíveis diante dos resultados encontrados nesta investigação é a de que a utilização da forma IRF do MMPI na população brasileira não alfabetizada, embora apresente-se como uma possibilidade rápida e prática de avaliação psicológica, requer procedimentos para maior simplificação da redação dos itens. Contudo, mais relevante e urgente é a necessidade de um extenso trabalho de padronização, a fim de evitar um processo de estigmatização que atribui ao atípico e não usual o caráter de psicopatológico e coloca a ciência a serviço de uma determinada ideologia político-social.

Finalizando, é importante acrescentar que a análise dos protocolos invalidados pela escala $F$ aponta uma tendência do grupo a obter resultados $T \geq 70$ nas escalas $P a, S c$ e $H s$, indo ao encontro das conclusões indicadas na literatura quanto à prevalência de transtornos psicossomáticos e esquizofrênicos em migrantes. As ponderações realizadas neste estudo quanto à confiabilidade dos resultados, alertam para a necessidade de cuidados redobrados na escolha de instrumentos de avaliação psicológica em investigações dessa natureza, principalmente quando se verifica a diversidade de técnicas, em termos de qualidade e valor científico, que caracteriza as pesquisas epidemiológicas.

\section{Referências}

Almeida Filho, N. (1985). Cultura e psicopatologia: revisão da literatura epidemiológica latino-americana. Jornal Brasileiro de Psiquiatria. 34, 357-364.

Almeida Filho, N. (1986). Migração e rótulo psiquiátrico: um estudo de caso. Jornal Brasileiro de Psiquiatria. 35, 231-240.

Almeida Filho, N. \& Bastos, S. B. (1982). Estudo caso controle da associação entre migração e desordens depressivas em mulheres. Jornal Brasileiro de Psiquiatria, 31, 25-29.

Blanchard, J. S. (1981). Readability of the MMPI. Perceptual and Motor Skills, 52, 985-986.

Chaimowicz, F. (1997) A saúde dos idosos brasileiros às vésperas do século XXI: problemas, projeções e alternativas. Revista de Saúde Pública, 31, 184-200.

Cochrane, R. \& Bal, S.S. (1987). Migration and schizophrenia: an examination of five hypotheses. Social Psychiatry, 22, 181-191.

Cunha, J.A.; Freitas, N.K. \& Raymundo, M.G. (1986). Psicodiagnóstico. Porto Alegre: Artes Médicas.
Dahlstrom, W.G. \& Welsh, G.S. (1968) An MMPI Handbook: A Guide to Use in Clinical Practice and Research. Minneapolis: The University of Minnesota Press.

Escobar, J.I. (1998) Immigration and mental health: Why are immigrants better off? Archives of General Psychiatry, 55, 781782.

Graham, J. R. (1987) MMPI: Guia Práctica (E.O. Oxford University Press Inc., Trad.) México, D.F: El Manual Moderno, S.A. de C.V. (Trabalho original publicado em 1977).

Hathaway, S.R. \& McKinley, J.C. (1971) Inventário Multifásico Minnesota de Personalidade - Manual (A. Benko \& R.J.P. Simões, Trads.) Rio de Janeiro: CEPA.

IBGE (1980) Censo demográfico: dados gerais, migração, instrução, fecundidade, mortalidade. Rio de Janeiro: IBGE, vol. 1 (19).

IBGE (1991) Censo demográfico. Rio de Janeiro: IBGE, $n^{\circ} 1$.

IBGE (1997) Contagem da população. Rio de Janeiro: IBGE, vol. 2.

Johanson, L.M., Sundquist, J., Johanson, S.E. \& Bergman, B. (1998) Immigration, moving house and psychiatric admissions. Acta Psychiatrica Scandinavica, 98, 105-111.

Johnson, R.H. \& Bond, G.L. (1950). Reading Ease of Commonly Used Tests. Journal of Applied Psychology, 34, 319-324.

Mendonça, M., Elliot, M.G., McNeill, J., Rodriguez, R. \& Zelkind, J. (1984). An MMPI - Based Behavior Descriptor / Personality Trait List. Journal of Personality Assesment, 48, 483-485.

Pichot, P. (1961) Teste dos Dominós (D 48) - Manual (Nick, E. Trad.) Rio de Janeiro: CEPA.

Raven, J.C. (1950) Test de Matrices Progressivas - Manual. Buenos Aires, Paidós.

Sam, D.L. \& Berry, J.W. (1995). Acculturative stress among young immigrants in Norway. Scandinavian Journal of Psychology, $36,10-24$.

Saraiva, P. (1961) Estudo Médico-Psicológico de Casos de Deterioração Mental. Tese de Doutorado. Belo Horizonte, Departamento de Clínica Psiquiátrica da Faculdade de Medicina, Universidade de Minas Gerais.

Souza, M.F..M. \& Silva, G.R.S. (1998) Risco de distúrbios psiquiátricos menores em área metropolitana na região Sudeste do Brasil. Revista de Saúde Pública, 32, 50-58.

Vega, W.A., Kolody, B., Hwang, J. \& Noble, A. (1993). Prevalence and magnitude of perinatal substance exposures in California. New England Journal of Medicine, 329, 800-859.

Ward, C.L. (1986). Estimation of MMPI Scale Scores From an Improved Readability Short Form. Journal of Clinical Psychology, 42, 602-604.

Ward, C.L. \& Meyers, R. (1984). The Utility of an Improved Readability Short Form of the MMPI with Elderly Male Patients. Journal of Clinical Psychology, 40, 997-1000.

Ward, C.L. \& Selby, R. B. (1980). An Abbreviation of MMPI with Increased Comprehensibility and Readability. Journal of Clinical Psychology, 36, 180-185.

Weil, P. \& Nick, E. (1971). O Potencial da Inteligência do Brasileiro. Rio de Janeiro: CEPA.

$$
\begin{array}{r}
\text { Recebido em 17.03.2000 } \\
\text { Primeira decisão editorial em 12.02.2001 } \\
\text { Versão final em 24.04.2001 } \\
\text { Aceito em 10.05.2001 }
\end{array}
$$

